\title{
Rethinking the Clinically Based Thresholds of TransCelerate BioPharma for Risk-Based Monitoring
}

Therapeutic Innovation \& Regulatory Science 2018, Vol. 52(5) 560-57। (C) The Author(s) 2018 Article reuse guidelines: sagepub.com/journals-permissions DOI: $10.1177 / 216847901773898$ | tirs.sagepub.com

\author{
Richard C. Zink, PhD ${ }^{1,2}$, Anastasia Dmitrienko ${ }^{3}$, \\ and Alex Dmitrienko, PhD $^{4}$
}

\begin{abstract}
Background: The quality of data from clinical trials has received a great deal of attention in recent years. Of central importance is the need to protect the well-being of study participants and maintain the integrity of final analysis results. However, traditional approaches to assess data quality have come under increased scrutiny as providing little benefit for the substantial cost. Numerous regulatory guidance documents and industry position papers have described risk-based approaches to identify quality and safety issues. In particular, the position paper of TransCelerate BioPharma recommends defining risk thresholds to assess safety and quality risks based on past clinical experience. This exercise can be extremely time-consuming, and the resulting thresholds may only be relevant to a particular therapeutic area, patient or clinical site population. In addition, predefined thresholds cannot account for safety or quality issues where the underlying rate of observing a particular problem may change over the course of a clinical trial, and often do not consider varying patient exposure. Methods: In this manuscript, we appropriate rules commonly utilized for funnel plots to define a traffic-light system for risk indicators based on statistical criteria that consider the duration of patient follow-up. Further, we describe how these methods can be adapted to assess changing risk over time. Finally, we illustrate numerous graphical approaches to summarize and communicate risk, and discuss hybrid clinical-statistical approaches to allow for the assessment of risk at sites with low patient enrollment. Results: We illustrate the aforementioned methodologies for a clinical trial in patients with schizophrenia. Conclusions: Funnel plots are a flexible graphical technique that can form the basis for a riskbased strategy to assess data integrity, while considering site sample size, patient exposure, and changing risk across time.
\end{abstract}

\section{Keywords}

Data visualization, exposure-adjusted, risk-based approaches, statistical monitoring, time windows

\section{Introduction}

The quality of data from clinical trials has received a great deal of attention in recent years. Of central importance is the need to protect the well-being of study participants and maintain the integrity of final analysis results, which is collectively referred to as Good Clinical Practice (GCP). ${ }^{1}$ In the past, monitoring activities frequently engaged in $100 \%$ source data verification (SDV) of respective case report forms during regular on-site visits to the clinical sites. However, the traditional approach to assess data quality for clinical trials in medical product development has come under increased scrutiny as providing little benefit for the substantial cost. In a study of 9 clinical trials from 6 of the member companies of TransCelerate Biopharma Inc, it was observed that SDV generated $7.8 \%$ and $2.4 \%$ of the total queries identified for data in all and critical fields, respectively. ${ }^{2}$ The remaining queries were identified through regular database cleaning activities. In a more recent study of 1168 phase I-IV trials across 53 sponsors, TransCelerate found that a median rate of $1.1 \%$ of all fields in electronic case report forms were corrected due to SDV. ${ }^{3}$ In addition, the authors performed a literature review of articles published between 2008 and 2014 to summarize individual assessments of SDV. For example, in an international multi-center trial of 9385 women studied for up to 2 years, Bakobaki and coauthors found that $95 \%$ of data findings were or could have been identified from the database alone. ${ }^{4}$

\footnotetext{
'JMP Life Sciences, SAS Institute Inc, Cary, NC, USA

${ }^{2}$ Department of Biostatistics, University of North Carolina at Chapel Hill, Chapel Hill, NC, USA

${ }^{3}$ Columbia University, New York, NY, USA

${ }^{4}$ Mediana Inc, Overland Park, KS, USA
}

Submitted 5-Sep-2017; accepted 25-Sep-2017

Corresponding Author:

Richard C. Zink, PhD, Senior Director, Data Management and Statistics, Target PharmaSolutions, Chapel Hill, NC, USA.

Email: richard.c.zink@gmail.com 


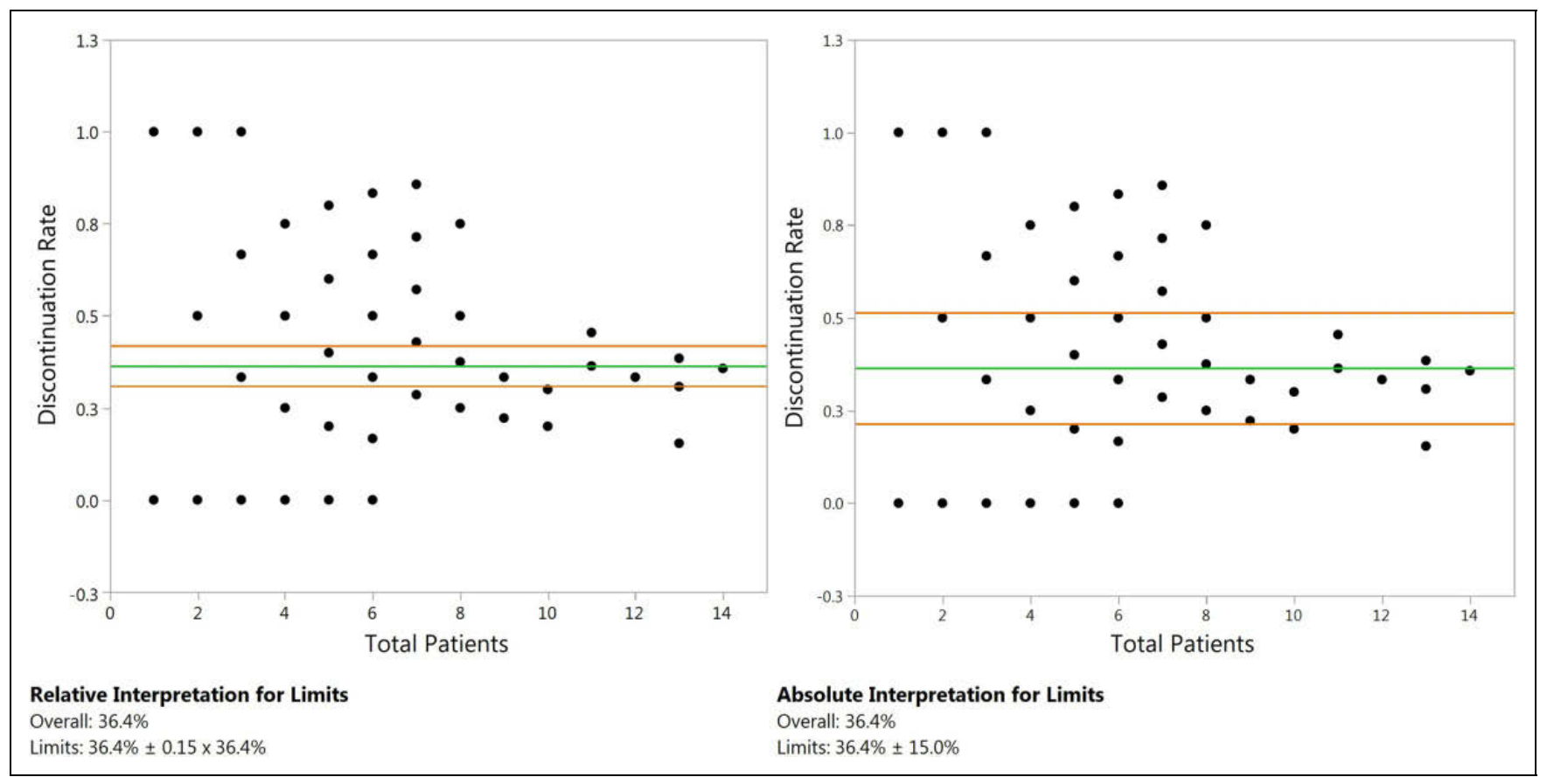

Figure I. Basic set of risk thresholds in a clinical trial with a binary event (early discontinuation from the trial). The risk thresholds are computed for the average discontinuation rate at each site, with an overall rate of discontinuation of $36.4 \%$. In the left panel, relative limits are displayed ( $31.0 \%$ and $41.9 \%)$. In the right panel, absolute limits are displayed (2I.4\% and $51.4 \%)$.

Numerous regulatory guidance documents and papers have described risk-based approaches to identify quality and safety issues using centralized monitoring of the study database employing data visualization, sampling techniques, and other statistical methodologies. ${ }^{2,5-13}$ In particular, the position paper of TransCelerate BioPharma recommends that sponsors define risk thresholds to assess safety and quality risks based on past clinical experience. Using an example from the appendix of the position paper, consider a clinical trial in which a sponsor wants to assess safety risk by using the average number of adverse events per patient to perform comparisons between the clinical sites. Risk thresholds are defined in the following manner:

- Mild: site average within 5\% of the overall average for all sites

- Moderate: site average greater than 5\% and within $10 \%$ of the overall average for all sites

- Severe: site average greater than $15 \%$ of the overall average for all sites

In a dashboard, risks can be colored green, yellow, or red for mild, moderate, and severe, respectively. From the position paper, it isn't entirely clear if the suggested thresholds are meant to be interpreted in absolute or relative terms to the observed overall site rate. However, whether the thresholds are to be viewed in absolute or relative terms should be clearly communicated, and the resulting thresholds under the selected approach should be clinically meaningful. We return to this point below.

Generating the above risk thresholds can be extremely timeconsuming, particularly when considering the performance of past trials and building consensus among the study team as to what is unacceptable to observe among sites in practice. The resulting set of thresholds may be relevant only to a particular therapeutic area, patient or clinical site population. Further, these risk thresholds, as defined, ignore the number of patients that have either been enrolled or randomized at the clinical sites. Regardless of the sample size, each site is compared to the same set of risk thresholds (Figure 1). For example, Figure 1 presents the discontinuation rates for 98 sites, with the same threshold (in excess of $\pm 15 \%$ of the overall discontinuation rate) applied to all sites. In the left panel, a relative interpretation is applied where limits are $\pm 15 \%$ of the overall discontinuation rate while the left panel displays an absolute interpretation where limits are $15 \%$ higher or lower than the observed rate. If we assume that site risks are sampled according to some assumed homogenous risk across the sites, the risk estimates from smaller sites are naturally more variable than those from larger sites. In other words, it is not unusual to see more extreme results for smaller sites. Further, risk thresholds that are predefined within an Integrated Quality and Risk Management Plan (IQRMP) cannot account for risks that may vary according to past experience, resulting in necessary changes, nor can they easily account for risks that may change over the course of a clinical trial. For example, the risk of certain AEs may reduce as patients develop tolerability to the study 


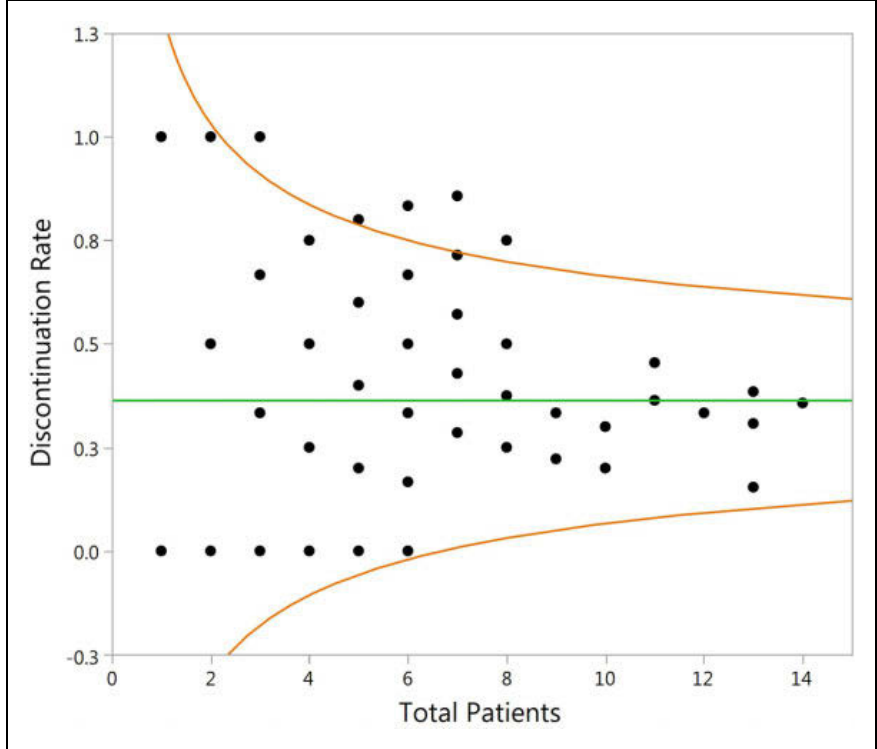

Figure 2. Risk thresholds based on a funnel plot in a clinical trial with a binary event (early discontinuation from the trial). The risk thresholds are based on a $95 \%$ confidence interval for the overall rate of discontinuation, varying sample size over the range of site sample sizes.

medications. Alternatively, greater exposure to drug may result in an increased likelihood of certain events. In addition, risk thresholds based on the site's sample size do not consider varying levels of patient exposure or follow-up between the sites.

In this paper, we appropriate rules commonly utilized for funnel plots to define a traffic-light system for risk indicators to assess data quality and safety. Funnel plots (Figure 2) display varying risk thresholds or control limits based on the sample sizes of the sites under investigation. ${ }^{14}$ The overall risk across sites is summarized as a vertical reference line, with control limits becoming narrower as the sample size increases along the $\mathrm{x}$-axis. From the funnel plot, we define a set of risk thresholds that consider the variation in either sample size or patient exposure among clinical sites. Further, we describe how multiple funnel plots can be used to assess changing risk over time. We illustrate the proposed methods for the rate of patient discontinuation from a clinical trial in patients with schizophrenia. Finally, we discuss hybrid clinical-statistical approaches to allow for the assessment of risk at sites with low patient enrollment.

\section{Funnel Plots Ignoring Patient Exposure}

\section{Binary Outcomes}

This section focuses on the most common scenarios in riskbased monitoring, namely, the cases when the sponsor is interested in monitoring binary events based on the event rate, allowing for a single event per patient, or event counts, allowing for multiple events per patient. Further, in each analysis described below, we consider that we are analyzing a snapshot of the clinical trial database, that is, that the number of sites, patients, or events are based entirely on what is currently available in the database. Consider the problem of monitoring binary outcomes, such as death or discontinuation from the study, across multiple sites in a clinical trial. Assume that there are $m$ sites with $n_{i}$ patients at the $i$ th site, $i=1,2, \ldots, m$. Denote the total number of patients in the study by $n=\sum_{i=1}^{m} n_{i}$. Let $X_{i j}$ signify the outcome of interest for the $j$ th patient at the $i$ th site, where $X_{i j}=1$ indicates that an event has occurred and $X_{i j}=0$ indicates that an event has not occurred. Finally, let $\rho_{i}$ denote the true event rate at the $i$ th site. Monitoring tools focus on testing the null hypothesis of consistency of the true event rates across the sites. Specifically, the null hypothesis states that the event rate is constant across the sites, that is, $H_{0}: \rho_{1}=\ldots=\rho_{m}=\rho$, where $\rho$ is the common event rate. This common event rate can be estimated as $\hat{\rho}=\frac{1}{n} \sum_{i=1}^{m} \sum_{j=1}^{n_{i}} X_{i j}$.

The control limits for the event rate at a particular site are defined as the $100(1-\alpha) \%$ confidence limits for the true event rate under the null hypothesis of consistency; a $95 \%$ confidence interval is obtained if $\alpha=0.05$. In other words, the confidence limits are computed under the assumption that the observed event rate at each site is $\hat{\rho}$. The resulting control limits quantify the amount of variability that would be expected at the site if the observed event rate was actually equal to $\hat{\rho}$.

We illustrate the process of computing the control limits using confidence limits based on an asymptotic normal approximation. Let $X_{i}=\sum_{j=1}^{n_{i}} X_{i j}$ represent the total number of events that occur and let $\hat{\rho}_{i}=X_{i} / n_{i}$ denote the estimated event rate at the $i$ th site. The asymptotic $100(1-\alpha) \%$ confidence interval for $\rho_{i}$ is given by

$$
\hat{\rho}_{i}-z_{1-\alpha / 2} \sqrt{\frac{\hat{\rho}_{i}\left(1-\hat{\rho}_{i}\right)}{n_{i}}} \leq \rho_{i} \leq \hat{\rho}_{i}+z_{1-\alpha / 2} \sqrt{\frac{\hat{\rho}_{i}\left(1-\hat{\rho}_{i}\right)}{n_{i}}}
$$

where $z_{1-\alpha / 2}$ is the upper percentile of the standard normal distribution. To construct the control limits for the observed event rate at this site, the estimated event rate is forced to be equal to the overall event rate $\hat{\rho}$. This means that the lower and upper asymptotic control limits for the $i$ th site are defined as $l_{i}=\hat{\rho}-z_{1-\alpha / 2} \sqrt{\frac{\hat{\rho}(1-\hat{\rho})}{n_{i}}}$ and $u_{i}=\hat{\rho}+z_{1-\alpha / 2} \sqrt{\frac{\hat{\rho}(1-\hat{\rho})}{n_{i}}}$, respectively. Asymptotic control limits may not be reliable in smaller clinical trials, so exact limits for an event rate may be preferable. For completeness, exact methods are summarized in the appendix.

\section{Count-Type Outcomes}

This section assumes that safety monitoring focuses on multiple events per patient. Count-type outcomes, such as the number of adverse events per patient, are commonly used to assess the safety profile at each site in a clinical trial. Using similar notation as above, here $X_{i j}$ is a count-type outcome variable for 
the $j$ th patient at the $i$ th site that follows a Poisson distribution. In particular, it is assumed that the outcomes at the $i$ th site follow a Poisson distribution with the parameter $\lambda_{i}$. This means that the average number of events as well as the variance of the number of events is equal to $\lambda_{i}$.

Control limits are defined for the average number of events per site. Here, we assume a constant time on study for all patients in the trial, so exposure is ignored when defining control limits. The parameter of interest in this setting is the average event count at each site, that is, $\lambda_{i}, i=1,2, \ldots, m$. Control limits are used to test the null hypothesis of consistency for this parameter. Under the null hypothesis, the average event counts are equal to the same value across the sites, that is, $H_{0}: \lambda_{1}=\ldots=\lambda_{m}=\lambda$, where $\lambda$ is the overall mean across sites. This parameter is estimated as $\hat{\lambda}=\frac{1}{n} \sum_{i=1}^{m} \sum_{j=1}^{n_{i}} X_{i j}$. Similarly, site-specific rates are defined as $\hat{\lambda}_{i}=\frac{X_{i}}{n_{i}}$, where $X_{i}=\sum_{j=1}^{n_{i}} X_{i j}$ is the number of events observed at the $i$ th site.

The asymptotic $100(1-\alpha) \%$ confidence interval for the mean number of events at the $i$ th site, that is, $\lambda_{i}$, is given by

$$
\hat{\lambda}_{i}-z_{1-\alpha / 2} \sqrt{\frac{\hat{\lambda}_{i}}{n_{i}}} \leq \rho_{i} \leq \hat{\lambda}_{i}+z_{1-\alpha / 2} \sqrt{\frac{\hat{\lambda}_{i}}{n_{i}}} .
$$

Under the null hypothesis of consistency, the site-specific means are equal to the overall mean and, thus, to define the control limits for the average number of events at the $i$ th site, the mean at this site is set to the overall mean. In other words, $\hat{\lambda}_{i}$ is replaced with $\hat{\lambda}$ and, as a consequence, the lower and upper asymptotic control limits are defined at the $i$ th site as $l_{i}=\hat{\lambda}-z_{1-\alpha / 2} \sqrt{\frac{\hat{\lambda}}{n_{i}}}$, and $u_{i}=\hat{\lambda}+z_{1-\alpha / 2} \sqrt{\frac{\hat{\lambda}}{n_{i}}}$. For completeness, an exact approach for count-type outcomes is summarized in the appendix.

\section{Applying Funnel Plots to the TransCelerate Framework}

The authors of the TransCelerate position paper on risk-based monitoring suggest that risks should be categorized and colored according to severity, with green, yellow, and red indicating mild, moderate, and severe risk, respectively. ${ }^{2}$ Funnel plots can be applied to this problem by defining multiple control limits, as is often done in practice. For example, $95 \%$ and $99.7 \%$ confidence intervals define a set of control limits in order to identify sites that exceed 2 (1.96, to be exact) and 3 standard errors from the overall estimate. Sites beyond the $99.7 \% \mathrm{lim}-$ its are flagged as having severe risk (red), and those beyond the $95 \%$ limits are flagged as having moderate risk (yellow); otherwise sites are considered to have mild risk and are colored green. It is worth pointing out that rules based on 3 standard deviations are commonly used in statistical process control to identify a manufacturing process that has gone "out of control." 15

If further granularity is required, additional control limits can be defined for describing risk. Additionally, risks can be considered in one direction only. For example, a sponsor more interested in safety could use the upper control limit to identify sites with an excess of adverse events, or a sponsor carefully monitoring patient enrollment may use the upper limit to identify sites that appear to be enrolling patients too quickly. Onesided control limits can be defined so that all of the type I error rate is used for either the lower or upper limit, which would provide greater power for identifying noteworthy signals in the direction of interest.

Unlike the risk thresholds suggested in Section 8.1.4 of the TransCelerate paper that are constant for all sites, the funnel plots control limits account for the varying sample size between clinical sites. Intuitively, it makes sense to consider varying site sample sizes in defining risk thresholds, since the estimates from smaller sites are more variable and, hence, less precise. Risk thresholds defined using funnels plots address this issue. However, the funnel plots as suggested are potentially flawed; they do not consider the varying exposure of patients within sites which would be common in most clinical trials. For example, suppose 2 sites have the same number of patients, with patients at sites A and B experiencing 6 and 24 events, respectively. Initially, we may conclude that site B may have excessive risk given the larger number of events. However, consider that the cumulative follow-up time for patients at site A is 3 months, while patients at site B have a total of 1 year of exposure. Despite the larger number of events at site B, each site observes 2 events per month, on average. This simple example shows the importance of considering patient exposure in assessing risk to avoid misleading results. We explore this issue in detail in the next section.

\section{Funnel Plots Accounting for Patient Exposure}

\section{Exposure Is Considered Fixed}

In this section, control limits are defined for the average number of events per site per unit of time. For a binary outcome $X_{i j}$, let $t_{i j}$ denote the exposure (time on study) for the $j$ th patient at the $i$ th site, where $t_{i j}$ is the time to the first event (if multiple are possible), or the last follow-up time for patients without an event of interest. Assume the events are independent and occur at a constant rate over time. A Poisson approximation is used to describe the distribution of the number of events over a specified period of time observed at each site, as in the exposure-adjusted incidence rate (EAIR). ${ }^{16}$ This approximation is appropriate when the event rate is low, say around $5 \%$, since this may imply a constant or nearly constant hazard rate over time. However, the EAIR may be preferred to the traditional approach since the crude proportion can be biased with highly variable follow-up time (eg, as seen in a study with at least 20\%-30\% of patients discontinuing), which will certainly be observed as data for an ongoing trial are evaluated for quality. Below, we will address the limitation for the constant hazard rate assumption. 
Assume that the number of events up to time $T$ is Poisson with mean $\lambda t$, where $\lambda$ is the event rate for a given unit of time. For the $i$ th site with $X_{i}=\sum_{j=1}^{n_{i}} X_{i j}$ events and $T_{i}=\sum_{j=1}^{n_{i}} t_{i j}$ exposure, define the EAIR as $\hat{\lambda}_{i}=\frac{X_{i}}{T_{i}}$. For all sites, define $X=\sum_{i=1}^{m} X_{i}$ and $T=\sum_{i=1}^{m} T_{i}$, with $\hat{\lambda}=\frac{X}{T}$. Under a normal approximation, a $(1-\alpha) \%$ confidence interval for the $i$ th site is $\hat{\lambda}_{i} \pm Z_{1-\frac{\alpha}{2}} \hat{\sigma}_{i}$, where $\hat{\sigma}_{i}=\sqrt{\frac{X_{i}}{T_{i}{ }^{2}}}=\sqrt{\frac{\hat{\lambda}_{i}}{T_{i}}}$. For these funnel plots accounting for exposure, the $\mathrm{x}$-axis representing the site sample size $(n)$ in the above examples is replaced by the total exposure time $T$. To develop a funnel plot, fix $\hat{\lambda}_{i}=\hat{\lambda}$, and vary $T$ from $\min \left(T_{i}\right)$ to $\max \left(T_{i}\right)$ to compute the control limits. As an area of future research, the work of Chan and Wang (2009) may suggest methods appropriate for computing an exact confidence interval for the EAIR. Finally, similar methods can be applied for a count-type endpoint $X_{i j}$, where $t_{i j}$ would denote the time on study for the $j$ th patient at the $i$ th site. ${ }^{17}$

\section{Exposure Is Considered Random}

In the previous section, we made the simplifying assumption that the patient-specific exposure $t_{i j}$ was fixed, ignoring any possible correlation between the frequency of events and patient exposure. In general, however, since patients enter the study at different times, and data integrity analyses will likely utilize data from ongoing patients, it is more appropriate to consider $t_{i j}$ as random. Here, assume $X_{i j}$ is the frequency of an event over a duration of $t_{i j}$ for the $j$ th patient at the $i$ th site. For the $i$ th site, an estimate of the number of events per unit time can be defined as $R_{i}=\frac{X_{i} / n_{i}}{T_{i} / n_{i}}{ }^{18}$ A corresponding estimate for this quantity over all sites is defined as $R=\frac{X}{T}$, where $X=\sum_{i=1}^{m} X_{i}$ and $T=\sum_{i=1}^{m} T_{i}$. We make no assumption about the joint distribution between $X_{i}$ and $T_{i}$, merely using the observed covariance matrix to calculate a variance for $R_{i}$.

Using the delta approximation, the variance of $R_{i}$ is estimated as

$$
\operatorname{Var}\left(R_{i}\right)=R_{i}^{2}\left(\frac{\operatorname{Var}\left(X_{i}\right)}{X_{i}^{2}}-\frac{2 \operatorname{Cov}\left(X_{i}, T_{i}\right)}{X_{i} T_{i}}+\frac{\operatorname{Var}\left(T_{i}\right)}{T_{i}^{2}}\right),
$$

where

$$
\begin{gathered}
\operatorname{Var}\left(X_{i}\right)=\frac{1}{n_{i}\left(n_{i}-1\right)} \sum_{j=1}^{n_{i}}\left(X_{i j}-\bar{X}_{i}\right)^{2}, \\
\operatorname{Var}\left(T_{i}\right)=\frac{1}{n_{i}\left(n_{i}-1\right)} \sum_{j=1}^{n_{i}}\left(T_{i j}-\bar{T}_{i}\right)^{2}, \\
\operatorname{Cov}\left(X_{i}, T_{i}\right)=\frac{1}{n_{i}\left(n_{i}-1\right)} \sum_{j=1}^{n_{i}}\left(X_{i j}-\bar{X}_{i}\right)\left(T_{i j}-\bar{T}_{i}\right), \\
\bar{X}_{i}=\frac{X_{i}}{n_{i}}, \\
\text { and } \bar{T}_{i}=\frac{T_{i}}{n_{i}} .
\end{gathered}
$$

To develop a funnel plot, fix $\hat{R}_{i}=\hat{R}$. To compute $\operatorname{Var}\left(R_{i}\right)$ at different exposures, the observed values for $T_{i}$ can be used to compute $X_{i}=\hat{R}_{i} T_{i}$ for the above formula, though such an approach will result in numerous values of $X_{i}$ that are noninteger.

\section{Allowing for Variable Risk Over Time}

Above, we have discussed alternate methods for accounting for varying patient exposure for binary and count-type outcomes. However, these initial suggestions are saddled with challenges that may limit their use in practice. Perhaps the biggest assumption, one that should be assessed in practice, is the constant hazard rate across time. Liu and coauthors suggest that this assumption is likely to hold for rare events, but this may not apply to many of the risk indicators that we want to assess for quality. ${ }^{16}$ Plus, it adds the additional burden of verifying an assumption in order for the analysis to be valid.

Given these limitations, one practical approach is to break up time into windows of fixed duration, say monthly or quarterly, and assess the risk separately within each window. First and foremost, this approach has the benefit of fixing the duration of time for all patients to the width of the predefined window, save for those patients who may happen to begin or discontinue the study at some point during the window. Analyses that ignore patient exposure may be appropriate to apply since exposure for most patients is fixed. A further benefit is that exact methods for analysis already exist (see the appendix) to generate funnel plots. However, for studies of larger size, the EAIR methods can be used to account for some variability in exposure for patients who enter or exit the study during a particular time window. Finally, breaking the study period into windows of fixed duration allows for differing hazard rates across time.

However, there are 2 ways to account for time as suggested in Zink. ${ }^{19}$ Windows can be defined according to the following scales:

1. Absolute: Time windows are defined according to calendar time (eg, month or quarter), starting with the first patient enrolled in the study. Comparisons performed here will suggest how well each site performs for any given month or quarter, though the within-trial "site experience" may vary from site to site. For quality metrics, sites that have just started participating in the trial may appear bad compared to sites that have resolved any issues since joining the study. For this time scale, it may be difficult to assess data from sites that begin the trial early until sufficient sites are participating and recruiting patients. If an intervention is applied to address a widespread safety or quality issue across the sites, the absolute scale can be used to assess performance before or after the intervention. Currently, data are likely to be assessed in practice using the absolute scale. 


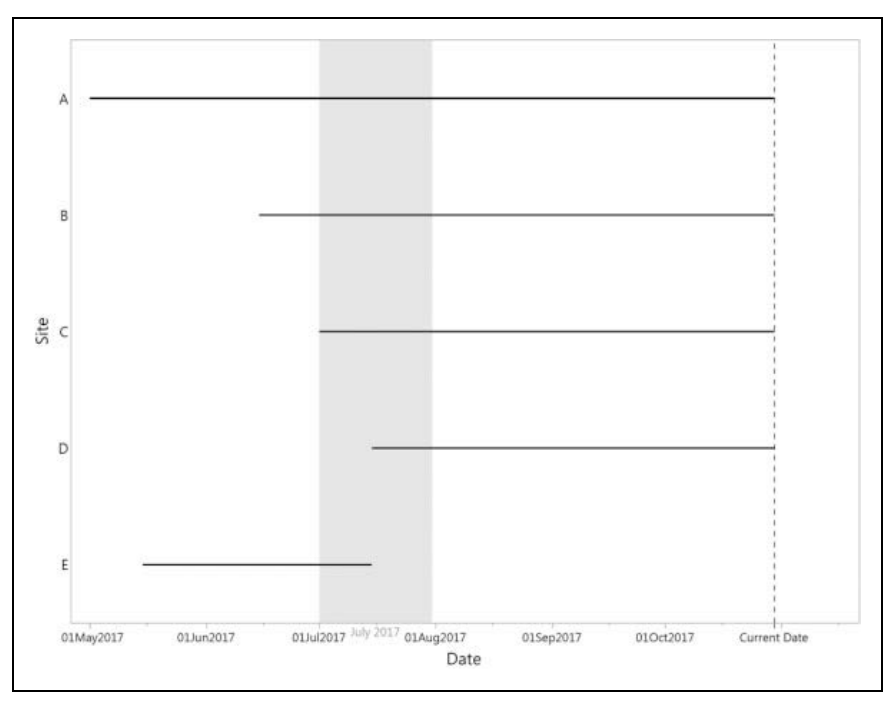

Figure 3. Analyzing time windows when considering time on the absolute scale, with July 2017 highlighted to illustrate differences in site experience and potential incomplete windows (sites $\mathrm{D}$ and $\mathrm{E}$ ).

2. Relative: Time windows are defined according to a within-site study day, with day 1 for a particular site being the first day that the site can begin enrolling patients, or the date the first patient is enrolled at the site. Comparisons performed here will suggest how well each site performs for a given amount of "site experience" within the current trial, since the data from the first month or quarter for each site will be compared, and so on. This approach allows for some comparison of windowed data for sites that participate early in the trial, though there may be limited data for later windows for sites with long durations of participation. If sponsor interventions are applied across the sites to address quality issues, analyses on the relative scale should be stratified according to major sponsor interventions, since these acts may occur at varying study days according to when each site began the trial.

Figure 3 presents an absolute time scale with a particular analysis interest in July 2017; site experience is measured from the beginning of each site line to the start of the shaded region. Here, site A clearly has more site experience than site B, and both of these sites have more experience than site $\mathrm{C}$ that has no experience prior to the July 2017 analysis window. Site D illustrates an incomplete window due to beginning the trial and/or enrolling the first patient later than the other sites. Site $\mathrm{E}$ has an incomplete window due to all ongoing patients either completing or discontinuing the trial. Figure 4 displays a relative time scale. Here, it will be possible to provide a within-trial assessment for the performance of site A during their first month; this would be challenging using the absolute scale. However, this figure illustrates that limited information may be available for later time windows.

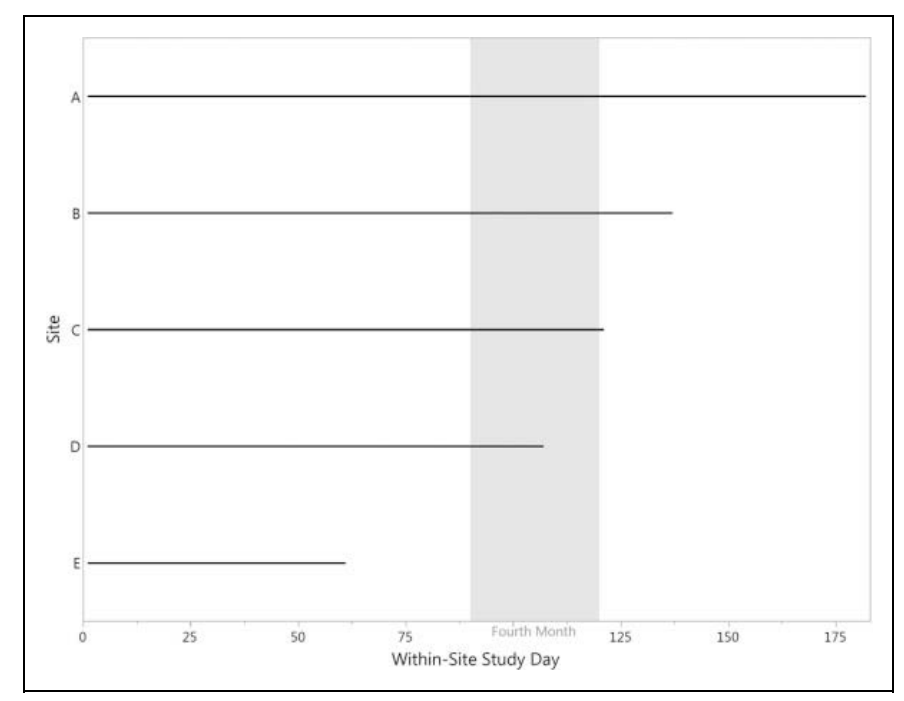

Figure 4. Analyzing time windows when considering time on the relative scale, with the fourth month highlighted to illustrate that later time windows may be incomplete (site D) or missed entirely (site E) for sites that participated in the trial more recently.

Analyses using either scale can be efficiently summarized using a heat map, where the y-axis would represent the site ID, the $\mathrm{x}$-axis would represent the time window, and the intersection of these 2 axes would be colored according to the risk observed. Each time window, or column along the x-axis in the heat map, would summarize the results from a funnel plot. Cells could be colored according to the traffic light color theme suggested by TransCelerate, or the direction above or below the center estimate could be incorporated into the color scheme. Alternatively, a continuous color scheme could be used based on observed risk indicator values centered by the overall estimate, and scaled by the standard error for the appropriate control limit based on the sample size or total exposure for each site. Examples of heat maps are provided in the Clinical Trial Example Section below.

\section{Multiplicity Considerations}

It is broadly recognized that the approaches to addressing multiplicity induced by the analysis of several efficacy endpoints in late-stage clinical trials become overly conservative when applied to multiplicity issues arising in the evaluation of safety signals or in the assessment of data quality. The standard approach to multiplicity adjustment in efficacy analyses relies on controlling the Type I error rate with respect to a family of predefined null hypotheses of no treatment effect. This error rate is defined as the probability of making at least one incorrect decision in the family, for example, erroneously rejecting at least one hypothesis that is known to be true. This definition is easily justified in the context of traditional multiplicity problems with a small number of hypotheses, say, up to 10 hypotheses. By contrast, multiplicity problems arising in safety and data quality assessments can be thought of as "massive" 
multiplicity problems since they are formulated in terms of hundreds and even thousands of hypotheses, for example, null hypotheses of no treatment effect on each group of adverse events in a clinical trial.

A more practical approach to tackling multiplicity in the context of risk-based monitoring can be set up by replacing the Type I error rate control with the false discovery rate control. ${ }^{20}$ Formally, the false discovery rate (FDR) is defined as the expected ratio of the number of rejected true null hypotheses to the total number of rejected hypotheses. In simple terms, using multiplicity adjustments aimed at controlling FDR is reasonable in settings where a certain proportion rather than a fixed number of errors is considered acceptable. FDR-controlling multiplicity adjustments in problems with a large number of safety variables have been recommended in Crowe et al. ${ }^{21}$ Several FDR-based methods have been proposed in the literature to develop an efficient strategy for handling multiplicity issues in the evaluation of clinical safety endpoints. ${ }^{22,23}$ As a quick illustration, the simple FDR-controlling test proposed in Benjamini and Hochberg $^{20}$ can be applied to funnel plots and TransCelerate signal detection. Assuming $k$ safety endpoints evaluated at $m$ sites (and possibly over $t$ time points), multiplicity-adjusted confidence intervals can be constructed based on the Benjamini-Hochberg test as follows. Let $P_{1}, P_{2}, \ldots, P_{k}$ denote the treatment effect $P$ values associated with the $k$ endpoints and $P_{(1)}<P_{(2)}<\cdots<P_{(k)} \quad$ denote the ordered $P$ values. The confidence intervals are defined using the adjusted level $\alpha^{*}=\frac{j}{k} \alpha$ for the largest index $j$ where $P_{(i)} \leq \frac{j}{k} \alpha$, where $\alpha$ is the regular level, for example, $\alpha=0.05$ (2-sided).

A potential limitation of simple FDR-based methods such as the Benjamini-Hochberg test is that the outcomes of interest, for example, safety variables, are assumed independent, which is not the case in a real clinical trial. An extended version of the Benjamini-Hochberg test, known as the Benjamini-HochbergYekutieli test, was developed for multiplicity problems with dependent outcomes. ${ }^{24}$ Further, resampling-based methods can be applied to perform multiplicity adjustments in settings with correlated outcomes. ${ }^{25}$ Resampling-based methods result in more efficient adjustments compared to simple FDR-based adjustments since the correlations among the outcomes of interest are explicitly taken into account. This approach has found numerous applications in other classes of massive multiplicity problems, including microarray experiments with hundreds of dependent variables. It is important to remember that resampling-based methods tend to be computationally intensive and thus may sometimes be less practical than straightforward adjustments based on the Benjamini-Hochberg or Benjamini-Hochberg-Yekutieli tests.

\section{Clinical Trial Example}

The approaches described above will be illustrated using an example based on a Phase III clinical trial in patients with schizophrenia. Adult patients with schizophrenia were enrolled at 98 sites to be randomly assigned to one of 3 doses of an experimental treatment or placebo (the sites were sequentially numbered 1 through 98). The early discontinuation rate tends to be quite high in clinical trials for the treatment of schizophrenia and can exceed $40 \%{ }^{26}$

Monitoring rules based on the TransCelerate approaches as well as the recommended approaches for funnel plots without considering exposure were applied to evaluate the extent of early discontinuation across the trial sites. The rules were applied to a blinded snapshot of the trial's database that was taken after 546 patients were enrolled in the trial. The number of patients per site in the resulting data set ranged between 1 and 14 . One hundred ninety-nine (36.4\%) patients dropped out of the trial in the selected data set. The appendix of the TransCelerate position paper contains suggested thresholds for patient discontinuation, which are reproduced here:

- Mild: site average within $5 \%$ to $15 \%$ of the overall rate for all sites and at least 3 subjects discontinued

- Moderate: site average greater than $15 \%$ and within $30 \%$ of the overall rate for all sites and at least 3 subjects discontinued

- Severe: site average greater than $30 \%$ of the overall rate for all sites and at least 4 subjects discontinued

The left panel of Figure 5 presents the monitoring rules based on the suggested TransCelerate risk thresholds summarized above according to an absolute interpretation of the thresholds. The overall reference is colored green, while reference lines are colored yellow or red to indicate moderate or severe thresholds. A key feature of the risk thresholds used in the left panel of Figure 5 is that they ignore the number of patients enrolled at each site. The thresholds tend to be overly conservative when applied to the sites with a small number of patients and may become too liberal as the number of patients per site increases. Many of the sites (37) are classified with either very low or very high patient discontinuation rates (severe, beyond the red thresholds). Forty-eight of 98 sites would be considered to have an acceptable discontinuation rate and would require no action if the TransCelerate monitoring rules were applied (between the yellow reference lines).

It is instructive to compare the monitoring rules depicted in the left and right panels of Figure 5. The right panel of Figure 5 shows the risk thresholds based on $95 \%$ (yellow) and $99.7 \%$ (red) confidence intervals for the binary event of interest (early discontinuation from the trial) when ignoring patient exposure. The risk thresholds were increasingly tighter as the number of patients per site increased. The early discontinuation rates were within the $95 \%$ confidence intervals at most of the sites. Six of 98 sites (sites 9, 53, 70, 75, 78, and 87) exhibited a moderately high discontinuation rate.

The risk thresholds displayed in the right panel of Figure 5 were derived using a simplifying assumption that the time on study was constant across the patients in the selected data set. 


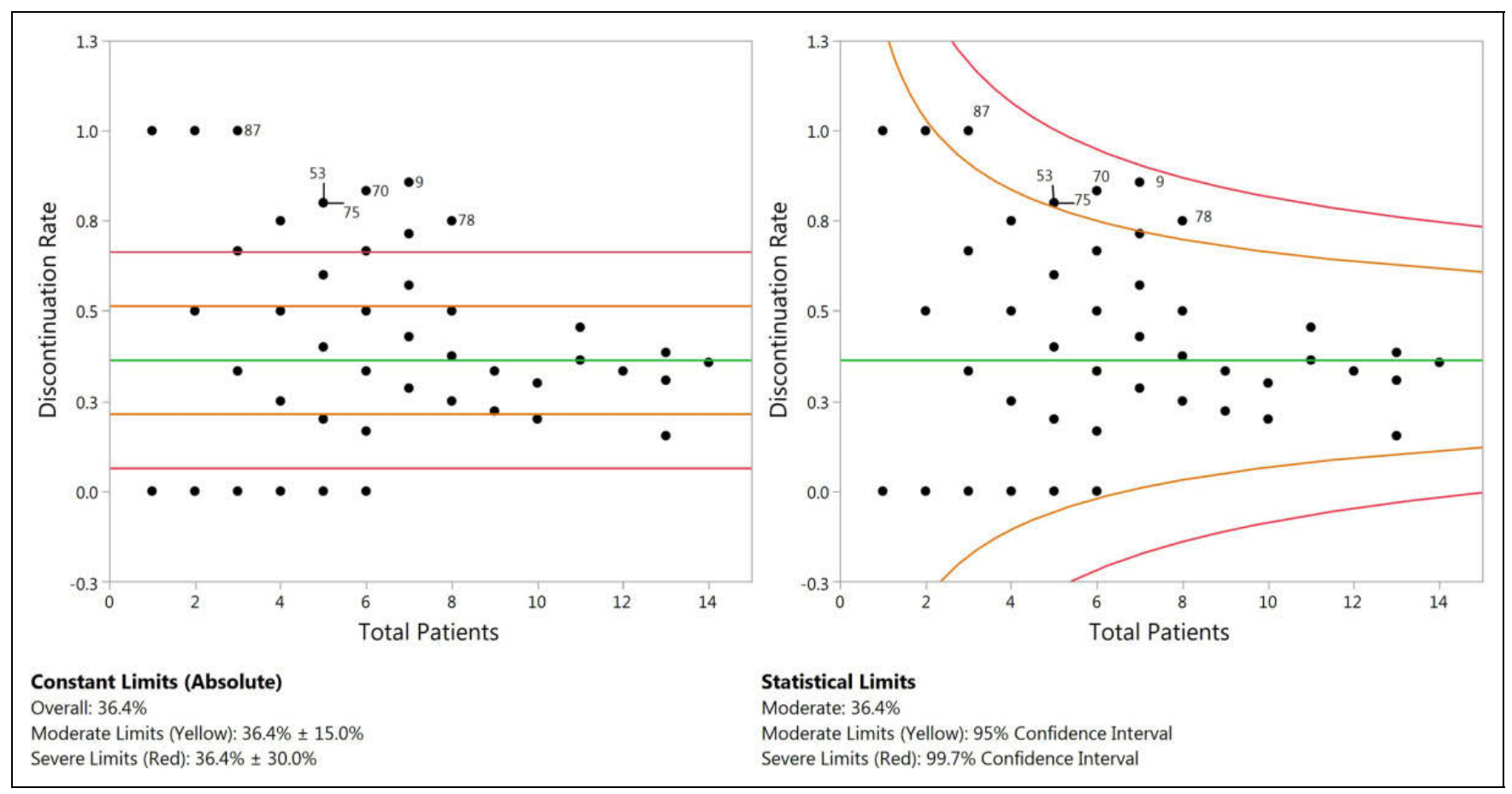

Figure 5. Risk thresholds based on TransCelerate rules in the schizophrenia clinical trial based on absolute an absolute interpretation (left) and a funnel plot (right). The overall rate of discontinuation was $36.4 \%$. In the left plot, moderate limits (yellow) are $21.4 \%$ and $51.4 \%$, and severe limits (red) are $21.4 \%$ and $66.4 \%$. The limits in the right plot are based on $95 \%$ (yellow) and $99.7 \%$ (red) confidence intervals.

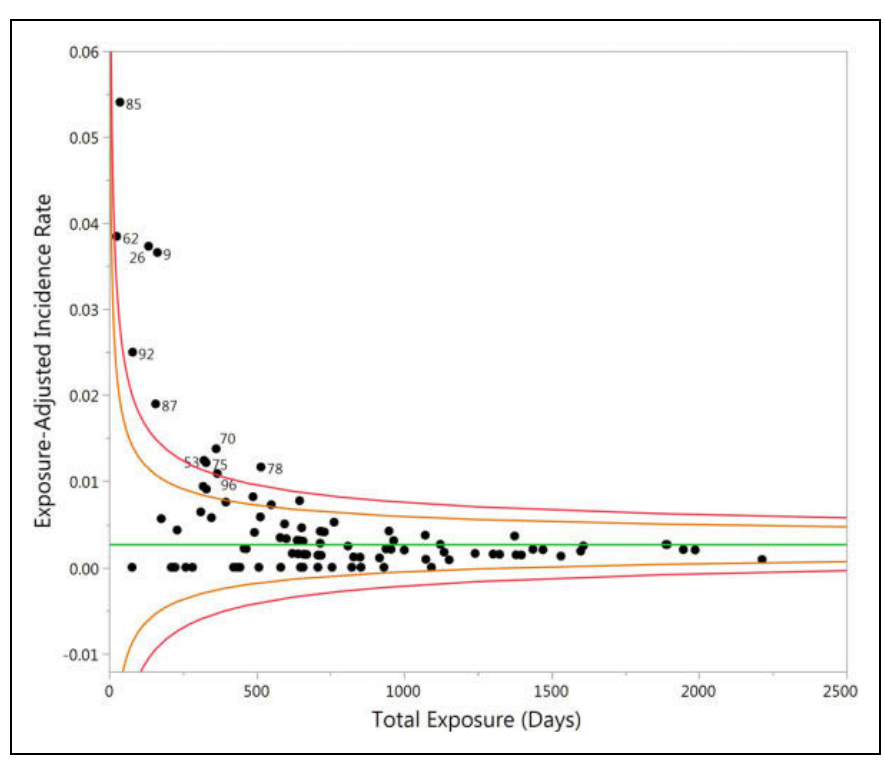

Figure 6. A funnel plot with risk thresholds based on $95 \%$ and $99.7 \%$ confidence intervals in the schizophrenia clinical trial. The risk thresholds are computed for the average discontinuation rate at each site and account for the duration of patient exposure.

In reality, there was much variability in the exposure times per patient, from 1 day to 252 days. To account for the variable exposure across patients from the trial sites, exposure-adjusted incidence rates were used to compute risk thresholds in Figure 6. This figure shows the resulting rate at each site along with the associated $95 \%$ and $99.7 \%$ confidence intervals. It follows from the figure that after this important adjustment for exposure, 5 additional sites can be viewed as outliers (sites 9 , $26,53,62,70,75,78,85,87,92$, and 96). It is worth noting that, for simplicity, patient exposure was assumed to be fixed in this figure and it will be quite straightforward to compute the $95 \%$ confidence intervals using the alternative approach based on the assumption of random exposure times.

Figures 7 and 8 present heat map-based summaries of early discontinuation rates across the 98 sites and 60-day time windows in the schizophrenia trial. The time windows were defined using the 2 approaches introduced in Section 3.3. The absolute time scale was used in Figure 7. The first patient was enrolled in the study on August 14, 1999, and the last patient's final visit included in the data snapshot occurred on December 24,2000 . Since the data snapshot used in this example was taken 499 days after the study start, nine 60-day time intervals were included in Figure 8. The relative time scale was used in Figure 8 (time windows were defined using the earliest withinsite start date). There are only 8 time windows in Figure 8 because 456 is the maximum within-site study day. Cells where no discontinuations were observed are de-emphasized in both figures in order to focus on sites and time windows where observed discontinuations were unusual. To use space more efficiently, only sites with at least 1 discontinuation are included in Figures 7 and 8.

The 2 heat maps provide an efficient way of examining the changes in the early discontinuation rates over time (it is 


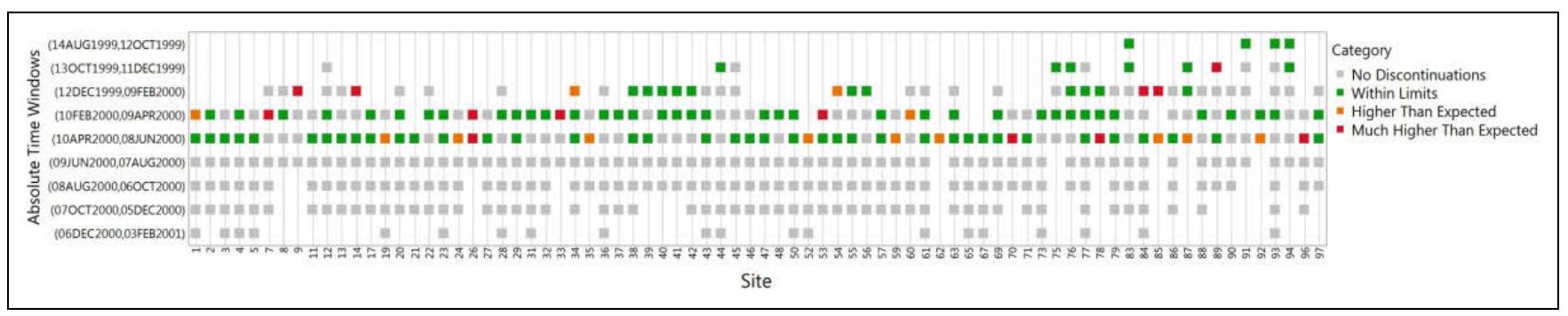

Figure 7. Heat map for the average discontinuation rate at each site within each of the 60-day time windows. The time windows are defined using the absolute time scale. Only sites with at least one discontinuation are included.

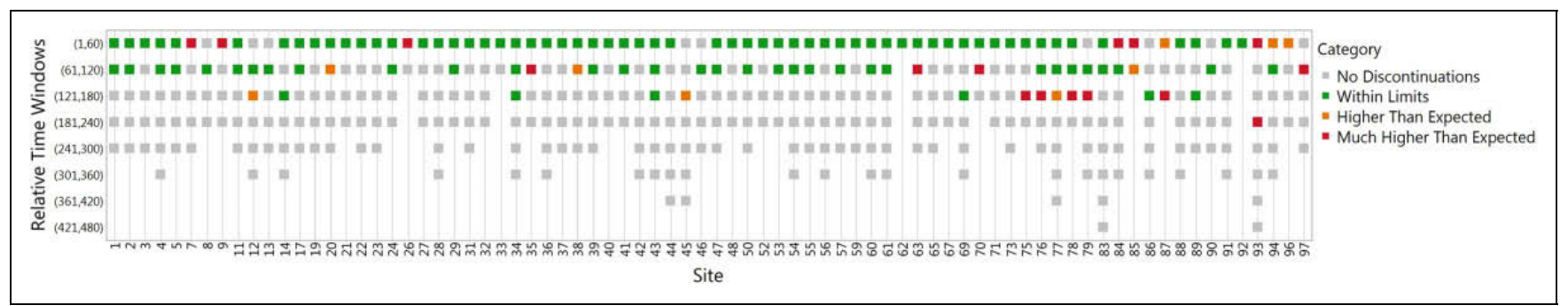

Figure 8. Heat map for the average discontinuation rate at each site within each of the 60-day time windows. The time windows are defined using the relative time scale. Only sites with at least one discontinuation are included.

worth noting that there were no discontinuations after June 4, 2000). In Figure 7, only site 26 had 2 periods in a row where there was a severe risk of discontinuation, and these periods were in the first 4 months that the site participated in the trial. If we examine site 26 in Figure 8, only the first time period shows elevated risk. How can this be interpreted? Clearly, under either time scale site 26 struggled during their first 60-day period in the trial. However, when comparing the second 60-day period to the other second 60-day periods of the other sites, site 26 appeared to be doing okay. The signal that arises on the absolute scale is likely due to the fact that the April 10 to June 8 period gave the sites that had already been participating in the trial for months the opportunity to refine their processes. Note that while site 26 shows up as a severe signal in Figures 6 to 8, it is noticeably absent from the right panel of Figure 5 where the impact of exposure is essentially ignored.

\section{Conclusions}

In lieu of the clinically based rules suggested by TransCelerate Biopharma for monitoring safety and quality data, funnel plots can be used to define a set of thresholds to identify quality and safety risks. ${ }^{2}$ The suggested approaches described above have a number of advantages. First, this strategy reduces the burden in defining a set of risk thresholds based on past experience before the start of a trial. Instead of providing customized thresholds for each risk indicator in the IQRMP, sponsors can make a blanket statement that control limits will be based on 1.96 and 3 standard errors for all indicators (with the possible addition of listing those indicators that will be evaluated in a single direction). In this way, sites are evaluated according to the data observed within the current study. Further, these thresholds can account for variations in sample size or patient exposure between the sites, features which are likely to occur in practice. Finally, defining time windows using absolute and relative time scales can allow for variations in the event rate for the events of interest across time. Not only will these windows give the sponsor an opportunity to assess site interventions, but they provide the needed flexibility for risks that may naturally change over time, such as when the clinical sites become more familiar with a protocol over the course of the study, or when a major protocol amendment has been approved. Since variations in patient exposure are limited within time windows, the exact methods summarized in the appendix can be utilized for trials of even modest size.

It is worth noting that TransCelerate has published recent papers that place greater emphasis on statistical methods to identify anomalies and characterize risk, though they report that in a recent survey at least one third of risk indicators rely on static nonstatistical thresholds. ${ }^{5-8}$ However, communicating statistical approaches, such as digit preference and Mahalanobis distance, to nonstatisticians can be challenging. The benefit of the proposed approach is that a variety of endpoints (eg, binary, count, normal [not shown]) and time considerations can be accommodated in the spirit of the original clinically based rules, and data visualization can be used to summarize and communicate risk. Further, the statistical thresholds can be presented according to the traffic light of the original TransCelerate position paper, which can further aid in the understanding of the approach. ${ }^{2}$ Of course, risk does 
not need to be confined to 3 levels (mild, moderate, and severe), and it would be straightforward under this framework to include additional risk thresholds as predefined multiples of the overall variability in the outcome to help prioritize which anomalies to address first.

Perhaps the biggest perceived downside of any statistical approach is that they may not be appropriate when site sample sizes are small. Regardless of the approach used, however, it is next to impossible to reliably assess safety or quality signals from a site with only 1 or 2 patients enrolled. In these cases, it may make sense to apply a hybrid clinical-statistical approach and apply the clinical criteria for sites with few patients, and the statistical criteria for sites of larger size. Alternatively, both criteria could be applied in conjunction, the clinical criteria identifying sites that are extreme compared to past experience, alongside statistical criteria that can highlight signals using control limits based on data in the current trial.

However, consider the following example for a binary outcome. Suppose, based on past experience, that $20 \%$ of patients are expected to discontinue a trial in a particular therapeutic area, and we define thresholds based on the TransCelerate suggestions as presented above, ignoring the limitation on sample size for now. A site discontinuation rate outside of $14 \%$ to $26 \%$ would be considered a severe risk. With 3 patients at a site, the possible responses are $0 \%, 33 \%, 67 \%$, and $100 \%$, all of which would be considered a severe risk. However, the observed sample size restrictions (at least 3 patients) prevents every instance from being interpreted as a signal, save for the situation where all patients discontinue (and this is only considered a moderate risk).

Table 1 summarizes the probability of observing various outcomes for varying site sample sizes of 1 to 6 patients with an assumed probability of discontinuation of 0.2 . A site with 3 patients will have all patients discontinue with a probability of 0.008. Similarly, a site with 4 patients will have all patients discontinue with a probability of 0.0016 . Admittedly, the latter result is rarer, which may be why a minimum of 4 is suggested in the position paper for a severe risk. However, a site with 4 of its 6 patients discontinuing exceeds the above clinical threshold for severe risk, even though this event is almost twice as likely (probability of 0.0154 ) to occur as a site with 3 patients all discontinuing the study! In short, this example illustrates that clinical criteria for risk thresholds may be problematic. Alternate rules may be required for sites with few patients, but these rules can and should be based on the probability of observing particular outcomes. Even when using probability, it is unlikely to identify risk at a site with 1 or 2 patients unless the particular outcome is rare. For therapeutic areas where these types of enrollment patterns are common, it may be necessary to analyze quality and safety retrospectively using the results from multiple trials. The methods of Koch et al can be used to extend the above methods by utilizing meta-analysis. ${ }^{18}$ Alternatively, Bayesian analyses can harness information from prior studies for binary and count-type outcomes by assuming that the true
Table I. Probability of Observing Discontinuations at a Site With Few Patients With a Probability of Discontinuing of 0.2 .

\begin{tabular}{|c|c|c|}
\hline Site Sample Size & Number of Discontinuations & Probability $^{\mathrm{a}}$ \\
\hline I & 0 & .8 \\
\hline I & I & .2 \\
\hline 2 & 0 & .64 \\
\hline 2 & 1 & .32 \\
\hline 2 & 2 & .04 \\
\hline 3 & 0 & .512 \\
\hline 3 & 1 & .384 \\
\hline 3 & 2 & .096 \\
\hline 3 & 3 & .008 \\
\hline 4 & 0 & .4096 \\
\hline 4 & 1 & .4096 \\
\hline 4 & 2 & .1536 \\
\hline 4 & 3 & .0256 \\
\hline 4 & 4 & .0016 \\
\hline 5 & 0 & .3277 \\
\hline 5 & I & .4096 \\
\hline 5 & 2 & .2048 \\
\hline 5 & 3 & .0512 \\
\hline 5 & 4 & .0064 \\
\hline 5 & 5 & .0003 \\
\hline 6 & 0 & .2621 \\
\hline 6 & 1 & .3932 \\
\hline 6 & 2 & .2458 \\
\hline 6 & 3 & .0819 \\
\hline 6 & 4 & .0154 \\
\hline 6 & 5 & .0015 \\
\hline 6 & 6 & .0001 \\
\hline
\end{tabular}

${ }^{\text {a }}$ Probabilities calculated using a binomial distribution.

event rate $\rho$ or the average number of events $\lambda$ can vary between sites according to a beta or gamma distribution, respectively. ${ }^{27}$ One final approach to consider is to group small sites that are local to one another so that if a signal is identified, a monitor can visit all sites during a single trip.

The original recommendations of TransCelerate may have been developed assuming that statisticians may not generally be available to provide their expertise toward the monitoring of clinical trial data. This is unfortunate, as statisticians possess a unique skill set ideal for uncovering and communicating risk. Statisticians should take an active interest in these pursuits, since any steps taken to improve data quality earlier in the timeline of a clinical trial contributes to more straightforward analysis and interpretation later on. It is comforting that subsequent research from TransCelerate has placed a greater emphasis on the usage of statistical methodologies in the determination of risk. However, statistics in isolation is not the answer to the problem of data quality. It is important to maintain a multidisciplinary approach to understand, identify, and address the risk in our clinical data. Methods need to be refined to identify anomalies that are of practical significance (think both clinically and statistically significant), and any signals will require some level of clinical experience in order to understand the importance of the findings. 


\section{Appendix}

\section{Exact Control Limits for a Binary Outcome lgnoring Patient Exposure}

Exact $100(1-\alpha) \%$ confidence limits for a proportion are defined as

$$
\begin{aligned}
l(X, n)=1 / & \left(1+\frac{n-X+1}{X} F_{2(n-X+1), 2 X, 1-\alpha / 2}\right), \\
u(X, n)= & \left(\frac{X+1}{n-X} F_{2(X+1), 2(n-X), 1-\alpha / 2}\right) / \\
& \left(1+\frac{X+1}{n-X} F_{2(X+1), 2(n-X), 1-\alpha / 2}\right),
\end{aligned}
$$

where $F_{k_{1}, k_{2}, 1-\alpha / 2}$ is the upper percentile of an $F$ distribution with $k_{1}$ and $k_{2}$ degrees of freedom. ${ }^{28}$ As above, to set up control limits based on an exact confidence interval, the estimated event rate at the $i$ th site needs to be set to the overall event rate. However, this may not be possible since $X_{i}=n_{i} \hat{\rho}$ may not be an integer. In practice, we utilize $X_{i *}=\left[n_{i} \hat{\rho}\right]$ and $X_{i}^{*}=\left[n_{i} \hat{\rho}\right]$, the largest integer less than or equal to $n_{i} \hat{\rho}$ and the smallest integer greater than or equal to $n_{i} \hat{\rho}$, respectively.

Two types of exact control limits can be constructed using these values. The first set of control limits, termed liberal control limits, are defined for the $i$ th site by plugging the lower bound for the number of events into the lower exact confidence limit and the upper bound into the upper exact confidence limit: $l_{i}=l\left(X_{i *}, n_{i}\right)$ and $u_{i}=u\left(X_{i}^{*}, n_{i}\right)$. By contrast, conservative control limits are constructed by using the upper bound for $X_{i}$ in the lower exact confidence limit and the lower bound for $X_{i}$ in the upper exact confidence limit: $l_{i}=l\left(X_{i}^{*}, n_{i}\right)$ and $u_{i}=u\left(X_{i *}, n_{i}\right)$. The conservative interval is guaranteed to be narrower than the liberal interval, making the former more appropriate for monitoring life-threatening events in clinical trials.

\section{Exact Control Limits for a Count-Type Outcome Ignoring Patient Exposure}

An exact approach relies on the exact confidence interval for the parameter of a Poisson distribution. ${ }^{29}$ The exact $100(1-\alpha) \%$ confidence interval for the mean number of events at the $i$ th site $\left(\lambda_{i}\right)$ is given by

$$
\begin{gathered}
l(X, n)=\frac{1}{2 n} \chi_{2(X+1), 1-\alpha / 2}^{2}, \\
u(X, n)=\frac{1}{2 n} \chi_{2 X, \alpha / 2}^{2},
\end{gathered}
$$

where $\chi_{k, 1-\alpha / 2}^{2}$ is the upper percentile of the chi-square distribution with $k$ degrees of freedom, and $\chi_{k, \alpha / 2}^{2}$ is the lower percentile of the chi-square distribution with $k$ degrees of freedom.

When setting up exact control limits for the mean number of events in a clinical trial, the trial's sponsor faces the same challenge as in the binary case. In particular, the control limits at each site need to be defined in such a way that the mean number of events at that site matches the overall mean. To accomplish this, the number of events at the $i$ th site $\left(r_{i}\right)$ needs to be chosen to satisfy $\hat{\lambda}=\frac{X_{i}}{n_{i}}$, which may not be possible since $X_{i}$ must be an integer. To overcome this, liberal and conservative control limits can be defined as in the case for binary outcomes.

\section{Authors' Note}

Since the time of this research, author Richard Zink has changed affiliation. He is now Senior Director, Data Management and Statistics, at Target PharmaSolutions, Chapel Hill, NC.

\section{Declaration of Conflicting Interests}

No potential conflicts were declared.

\section{Funding}

No financial support of the research, authorship, and/or publication of this article was declared.

\section{References}

1. International Conference of Harmonisation. E6: Guideline for Good Clinical Practice. http://www.ich.org/fileadmin/Public_ Web_Site/ICH_Products/Guidelines/Efficacy/E6_R1/Step4/E6_ R1_Guideline.pdf. Published 1996.

2. TransCelerate Biopharma Inc. Position paper: risk-based monitoring methodology. http:/www.transceleratebiopharmainc.com/ assets/risk-based-monitoring/. Published 2013.

3. Sheetz N, Wilson B, Benedict J, et al. Evaluating source data verification as a quality control measure in clinical trials. Therapeutic Innovation \& Regulatory Science. 2014;48:671-680.

4. Bakobaki JM, Rauchenberger M, Joffe N, McCormack S, Stenning S, Meredith S. The potential for central monitoring techniques to replace on-site monitoring: findings from an international multi centre clinical trial. Clin Trials. 2012;9:257-264.

5. Wilson B, Provencher T, Gough J, et al. Defining a central monitoring capability: sharing the experience of TransCelerate BioPharma's approach, part 1. Therapeutic Innovation \& Regulatory Science. 2014;48:529-535.

6. Gough J, Wilson B, Zerola M, et al. Defining a central monitoring capability: sharing the experience of TransCelerate BioPharma's approach, part 2. Therapeutic Innovation \& Regulatory Science. 2015;50:8-14.

7. Knepper D, Fenske C, Nadolny P, et al. Detecting data quality issues in clinical trials: current practices and recommendations. Therapeutic Innovation \& Regulatory Science. 2015;50:15-21.

8. Knepper D, Lindblad AS, Sharma G, et al. Statistical monitoring in clinical trials: best practices for detecting data anomalies suggestive of fabrication or misconduct. Therapeutic Innovation \& Regulatory Science. 2016;50:144-154.

9. US Food \& Drug Administration. (2013). Guidance for industry: Oversight of clinical investigations - a risk-based approach to monitoring. 
10. European Medicines Agency. Reflection paper on risk-based quality management in clinical trials. http://www.ema.euro pa.eu/docs/en_GB/document_library/Scientific_guideline/2013/ 11/WC500155491.pdf. Published 2013.

11. Buyse M, George SL, Evans S, et al. The role of biostatistics in the prevention, detection and treatment of fraud in clinical trials. Stat Med. 1999;18:3435-3451.

12. Venet D, Doffagne E, Burzykowski T, et al. A statistical approach to central monitoring of data quality in clinical trials. Clin Trials. 2012;9:705-713.

13. Zink RC. Risk-Based Monitoring and Fraud Detection in Clinical Trials Using JMP ${ }^{\circledR}$ and SAS ${ }^{\circledR}$. Cary, NC: SAS Institute Inc; 2014.

14. Spiegelhalter DJ. Funnel plots for comparing institutional performance. Stat Med. 2005;24:1185-1202.

15. Wheeler DJ. Advanced Topics in Statistical Process Control. Knoxville, TN: SPC Press; 2004.

16. Liu GF, Wang J, Liu K, Snavely DB. Confidence intervals for an exposure adjusted incidence rate difference with applications to clinical trials. Stat Med. 2006;25:1275-1286.

17. Chan I, Wang WWB. On analysis of the difference of two exposure-adjusted Poisson rates with stratification: from asymptotic to exact approaches. Stat Biosci. 2009;1:65-79.

18. Koch GG, Schmid JE, Begun JM, Maier WC. Meta-analysis of drug safety data. In: Gilbert GS, ed. Drug Safety Assessment in Clinical Trials. New York, NY: Marcel Dekker.

19. Zink RC. Exploring the challenges, impacts and implications of risk-based monitoring. Clin Investig. 2014;4:785-789.
20. Benjamini Y, Hochberg Y. Controlling the false discovery rate: a practical and powerful approach to multiple testing. J R Stat Soc B. 1995;57:289-300.

21. Crowe BJ, Xia HA, Berlin JA, et al. Recommendations for safety planning, data collection, evaluation and reporting during drug, biologic and vaccine development: a report of the safety planning, evaluation, and reporting team. Clin Trials. 2009;6:430-440.

22. Mehrotra DV, Heyse JF. Use of the false discovery rate for evaluating clinical safety data. Stat Methods Med Res. 2004;13:227-238.

23. Mehrotra DV, Adewale AJ. Flagging clinical adverse experiences: reducing false discoveries without materially compromising power for detecting true signals. Stat Med. 2012;31:1918-1930.

24. Benjamini Y, Yekutieli D. The control of the false discovery rate in multiple testing under dependency. Ann Statist. 2005;29:1165-1188.

25. Westfall PH, Young SS. Resampling-Based Multiple Testing: Examples and Methods for P-Value Adjustment. New York, NY: John Wiley \& Sons; 1993.

26. Correll CU, Skuban A, Ouyang J, et al. Efficacy and safety of brexpiprazole for the treatment of acute schizophrenia: a 6-week randomized, double-blind, placebo-controlled trial. Am J Psychiatry. 2015;172:870-880.

27. Gelman A, Carlin JB, Stern HS. Bayesian Data Analysis. 3rd ed. Boca Raton, FL: CRC Press; 2013.

28. Blyth CR. Approximate binomial confidence limits. J Am Statist Assoc. 1986;81:843-855.

29. Ulm K. A simple method to calculate the confidence interval of a standardized mortality ratio. Am J Epidemiol. 1990;131:373-375. 